\title{
Widely Tunable Terahertz Phase Modulation with Gate-Controlled Graphene Metasurfaces
}

\author{
Ziqi Miao, ${ }^{1}$ Qiong Wu, ${ }^{1}$ Xin Li, ${ }^{1}$ Qiong He, ${ }^{1,2}$ Kun Ding, ${ }^{1}$ Zhenghua An, ${ }^{1,2}$ Yuanbo Zhang, ${ }^{1,2, *}$ and Lei Zhou ${ }^{1,2, \dagger}$ \\ ${ }^{1}$ State Key Laboratory of Surface Physics, Key Laboratory of Micro \\ and Nano Photonic Structures (Ministry of Education), \\ and Department of Physics, Fudan University, Shanghai 200433, China \\ ${ }^{2}$ Collaborative Innovation Center of Advanced Microstructures, \\ Fudan University, Shanghai 200433, China \\ (Received 7 April 2015; published 16 November 2015)
}

\begin{abstract}
As the basis of a diverse set of photonic applications, such as hologram imaging, polarization, and wave front manipulation, the local phase control of electromagnetic waves is fundamental in photonic research. However, currently available bulky, passive, range-limited phase modulators pose an obstacle in photonic applications. Here, we propose a new mechanism to achieve a wide phase modulation range, with graphene used as a tunable loss to drive an underdamped to overdamped resonator transition. Based on this mechanism, we present widely tunable phase modulation in the terahertz regime, realized in gate-tuned ultrathin reflective graphene metasurfaces. A one-port resonator model, supported by full-wave simulations, explains the underlying physics of the discovered extreme phase modulation and indicates general strategies for designing tunable photonic devices. As an example, we demonstrate a gate-tunable terahertz (THz) polarization modulator with a graphene metasurface. Our findings establish the possibility for photonic applications based on active phase manipulation.
\end{abstract}

DOI: 10.1103/PhysRevX.5.041027

Subject Areas: Graphene, Metamaterials

The phase modulation of electromagnetic (EM) waves is crucial in photonics research. This is best exemplified by the Huygens principle: A far-field EM wave front is essentially determined by the phase distribution in a given near-field plane [1]. The ability to control the local EM phase has led to relevant photonic applications such as holographic imaging [2-6], polarization manipulations [7], and wave front control [8-10]. Such a phase modulation was conventionally achieved by modulating the refractive index of bulk materials (including both natural materials [11] and recently studied metamaterials) [12-15]. The dimensions of such systems are typically on the order of working wavelength $\lambda[2,11]$ and therefore too bulky for optical integration. Metasurfaces, which are ultrathin metamaterials consisting of planar subwavelength units, have been used to achieve phase modulations covering the full range of $360^{\circ}[9,10,16-19]$. However, currently available metasurface-based phase modulators are mostly passive elements that cannot be tuned externally. Although gatecontrolled graphene has been employed to tune the resonance in photonic systems, previous efforts have either focused solely on amplitude modulations [20-36] or resulted in a relatively narrow phase-tuning range $[37,38]$.

\footnotetext{
*zhyb@fudan.edu.cn

†phzhou@fudan.edu.cn
}

Published by the American Physical Society under the terms of the Creative Commons Attribution 3.0 License. Further distribution of this work must maintain attribution to the author(s) and the published article's title, journal citation, and DOI.
In this paper, we propose a new mechanism to significantly enlarge the phase-modulation range and demonstrate its capability in an ultrathin metasystem (thickness $\approx \lambda / 10$ ) with gate-tuned graphene integrated. We show that a gate bias applied to graphene through an ion liquid tunes its optical conductivity, transforms the coupled system from an underdamped resonator to an overdamped one, and drastically modulates the phase of the reflected wave. Within the coupled-mode theory (CMT) [39] framework, we develop an analytical model that captures the essence of our phasemodulation mechanism: a one-port resonator-a resonator with only one reflection channel—can drive the phase of the reflected wave across a $\pm 180^{\circ}$ transition when the losses in the resonator are fine-tuned (in our case, by graphene). This is in stark contrast to the two-port resonator (with both reflection and transmission channels) commonly used in previous studies $[37,38,40,41]$, in which only a small phase modulation has been possible. The proposed method suggests new design strategies for future active phase modulators. As an application of our findings, we present the experimental realization of a tunable $\mathrm{THz}$ polarization modulator based on our gate-controlled graphene metasurface.

The structure of our graphene metasurface is schematically shown in Fig. 1(a). The metasurface is a five-layer structure that we fabricate sequentially, starting from the bottom layer. An $\mathrm{Al}$ film is first evaporated onto a $\mathrm{SiO}_{2} / \mathrm{Si}$ substrate [not shown in Fig. 1(a)], and the film serves as a completely reflective surface for incident $\mathrm{THz}$ waves. Subsequently, we coat a layer of cross-linked photoresist SU8 (MicroChem), and then fabricate an array of Al mesas 

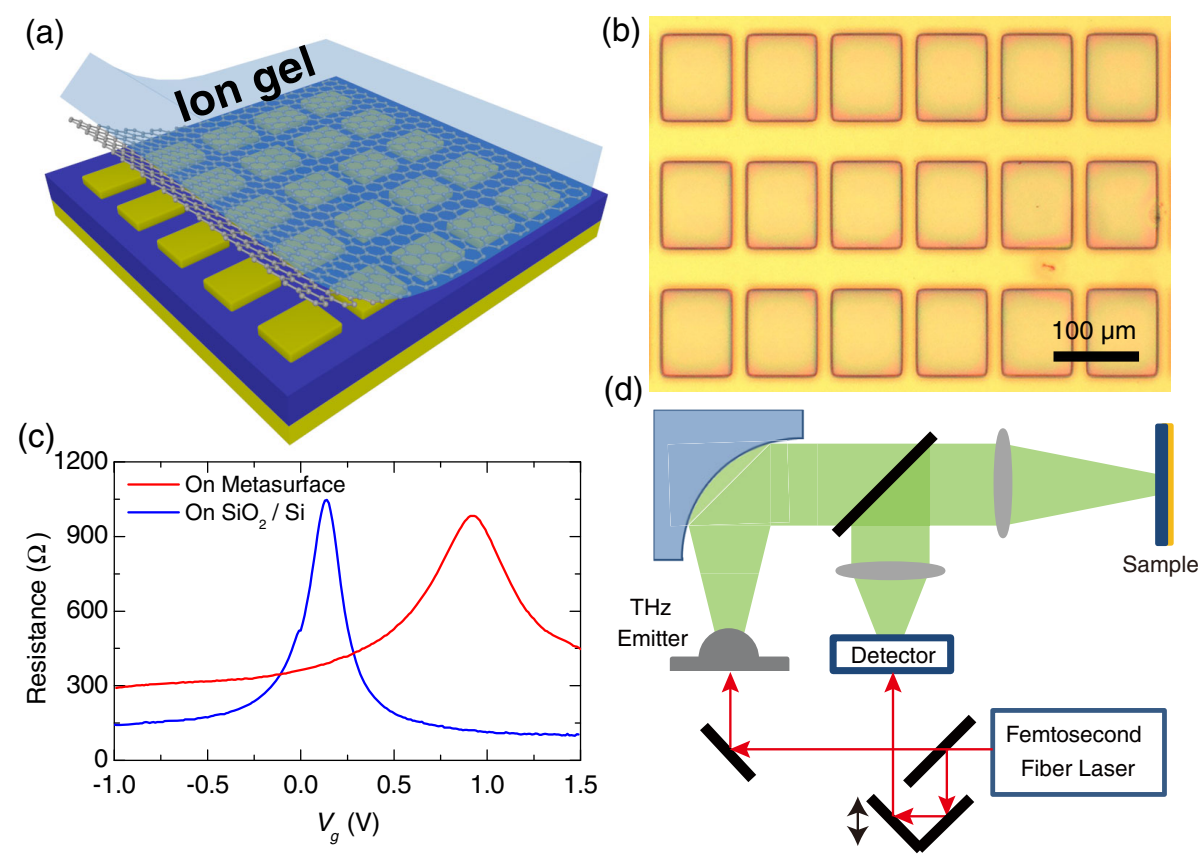

FIG. 1. Device geometry and experimental setup. (a) A schematic of the structure of the proposed device. The metasurface consists of an array of Al mesas and a continuous Al film (yellow) separated by an SU8 spacer (blue). CVD-grown monolayer graphene is transferred to the top of the metasurface and subsequently covered by a layer of gel-like ion liquid. The gate voltage applied between graphene and a top gate (not shown) controls the doping level of graphene. (b) An optical image of a typical device viewed from the top. (c) The gate-dependent resistance of CVD-grown graphene transferred to a silicon wafer (blue curve) and the metasurface (red curve).

(d) A schematic of the $\mathrm{THz}$ time-domain spectroscopy measurement system.

[Fig. 1(b)] on the layer by using standard optical lithography. The Al plane and the mesa array (both with a thickness of approximately $50 \mathrm{~nm}$ ) form a magnetic resonator because a circulating ac current is induced by a normally incident $\mathrm{THz}$ wave; the magnetic resonator is the focus of the current study $[42,43]$. The SU8 layer serves as a spacer between the $\mathrm{Al}$ plane and the mesa, and its thickness determines the coupling between the Al plane and the mesa. Finally, a layer of graphene is transferred onto the structure and subsequently covered by a layer of gel-like ion liquid. A gate bias $V_{g}$ applied between the graphene layer and a top gate is used to tune the resistance of graphene [26] [the peak resistance corresponds to the charge-neutral Dirac point $V_{D}$, as shown in Fig. 1(c)] and to modulate the loss of the resonator. The Dirac point of graphene shifts to $V_{D} \approx 1 \mathrm{~V}$ once it is transferred onto the metasurface. The shift is most likely induced by the charged impurities present around the graphene, and it does not affect our experiment. (Details of the device fabrication and characterization are discussed in Supplemental Material [44].) Once the devices are fabricated, we use THz time-domain spectroscopy to measure their reflection spectra for both amplitude and phase in a setup schematically depicted in Fig. 1(d). The thickness of the functional part of the device (excluding the optional $\mathrm{SiO}_{2} / \mathrm{Si}$ substrate) is on the order of $\lambda / 10$.

A variation in the graphene resistance, associated with the gate, drastically changes the resonance behavior of the proposed metasurface, leading to a phase modulation of $\pm 180^{\circ}$. This is demonstrated on a device with an $85-\mu$ m-thick SU8 layer, with the incident $\mathrm{THz}$ wave polarized along the long axis of the $\mathrm{Al}$ mesas (100 $\mu \mathrm{m} \times 160 \mu \mathrm{m}$ rectangles). The reflectance and associated phase spectra for varying gate voltages are shown in Figs. 2(a) and 2(b) and Figs. 2(c) and 2(d), respectively. The gate voltage relative to the Dirac point $\Delta V_{g}$ $\left(\Delta V_{g}=V_{g}-V_{D}\right)$ determines the doping level of graphene, and we use the nearly featureless spectrum at the highest doping level $\left(\Delta V_{g}=2.02 \mathrm{~V}\right)$ as the reference. At the Dirac point $\left(\Delta V_{g}=0\right)$, where graphene has the maximum resistance (and therefore, a minimal effect on the optical response of the underlying structure), we observe a resonance dip in the reflectance at $f=0.31 \mathrm{THz}$, and the phase shows a continuous $360^{\circ}$ variation across the resonance frequency: both observations are typical of the intrinsic behavior of a magnetic resonator $[7,42]$. As the doping level starts to increase from zero on the electron side $\left[\Delta V_{g}>0\right.$, Fig. 2(a)], the reflectance at resonance drops continuously. The resonator maintains an overall magnetic response with the same $360^{\circ}$ phase variation, although the bandwidth narrows [Fig. 2(c)]. However, a further increase in the gate bias beyond a critical voltage $\left(\Delta V_{C}=0.76 \mathrm{~V}\right)$ fundamentally changes the behavior of the resonator; the reflectance at resonance starts to increase as $\Delta V_{g}$ increases [Fig. 2(b)]. Notably, the 

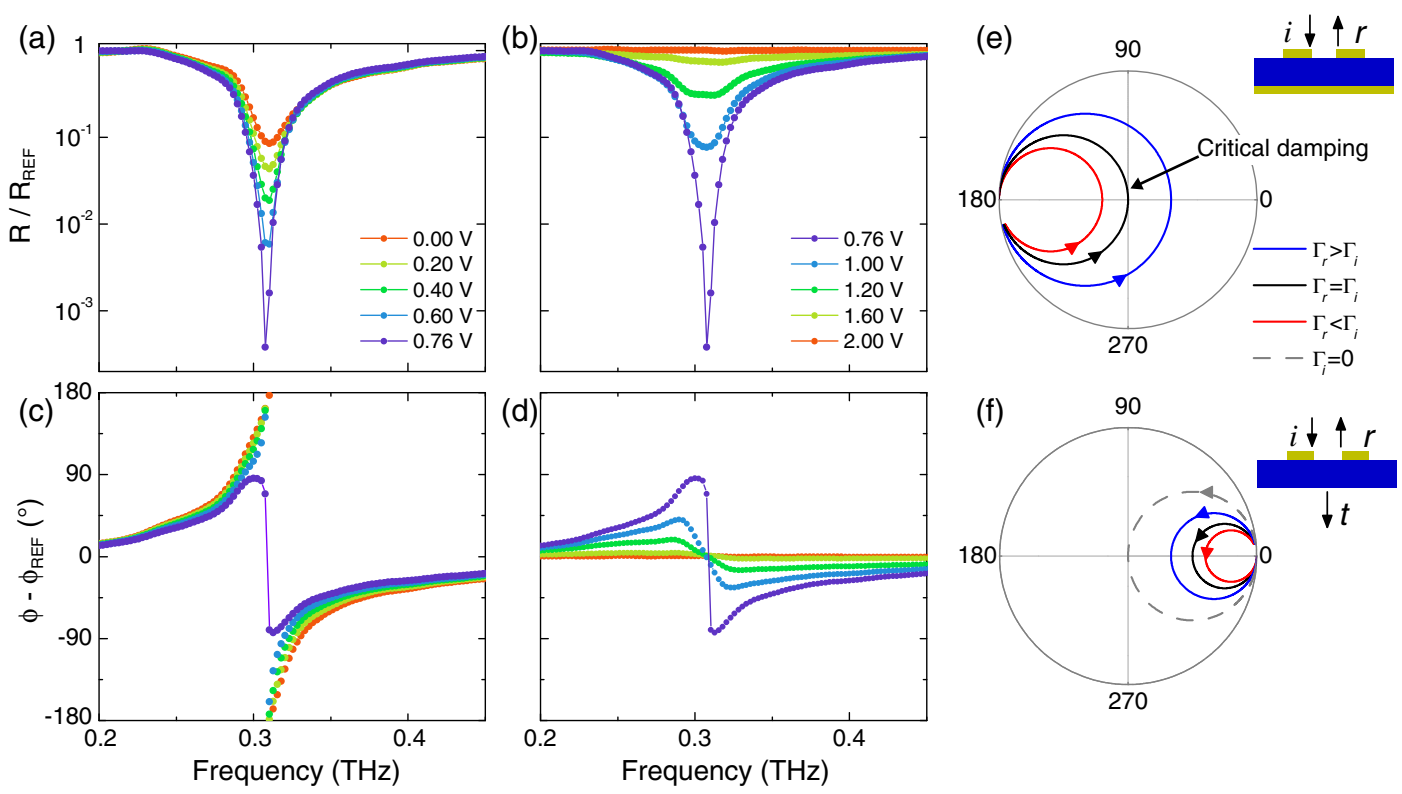

FIG. 2. Gate-controlled reflection modulation and the proposed resonator model based on CMT. The variation of reflectance $\left(R=|r|^{2}\right)$ with the relative gate voltage $\Delta V_{g}$. A critical transition occurs at $\Delta V_{c}=0.76 \mathrm{~V}$ (see text). The reflectances for $\Delta V_{g} \leq \Delta V_{c}$ and $\Delta V_{g} \geq \Delta V_{c}$ are shown in (a) and (b), respectively. The spectra are measured for a device with an 85 - $\mu$ m-thick SU8 spacer. The lateral dimensions of the Al mesas are $100 \mu \mathrm{m} \times 160 \mu \mathrm{m}$, and the mesa array has periods of $240 \mu \mathrm{m}$ in the $x$ and $y$ directions, respectively. The spectrum measured at $\Delta V_{g}=2.02 \mathrm{~V}$ is used as the reference. (c),(d) Phase modulations for $\Delta V_{g} \leq \Delta V_{c}$ and $\Delta V_{g} \geq \Delta V_{c}$, respectively. Data are obtained for the same device used for measurements in (a) and (b). (e) Smith curves of the reflection coefficient $r$ for the one-port model illustrated in the inset. The blue, black, and red curves represent underdamping, critical damping, and overdamping behavior, respectively. (f) Smith curves of the transmission coefficient $t$ for the two-port resonator model (inset). No critical transition is present, even in the limiting case $\Gamma_{i}=0$ (broken line).

phase spectrum stops showing a magnetic-resonance characteristic, but exhibits a decreasing phase variation centered at $0^{\circ}$. Our results clearly demonstrate an absolute phase modulation of $\pm 180^{\circ}$ associated with the gate at frequencies around the resonance, and, more importantly, they indicate a critical transition at $\Delta V_{g}=\Delta V_{C}$ in our metasystem. Such a transition is shown clearly in Figs. 4(a) and 4(d), where the critical transition points are seen in the reflectance and phase, both of which are measured as functions of the gate voltage and frequency. The resonator shows similar critical behavior on the hole side of the doping $\left(\Delta V_{g}<0\right)$; a detailed discussion is presented in Supplemental Materials [44].

The maximum phase modulation achieved using the present scheme is not limited to $180^{\circ}$; a much larger phase modulation can be achieved using two slightly different graphene metasurfaces gated independently [elements of devices A and B are illustrated in Figs. 3(a) and 3(b), respectively]. The large phase modulation is possible because gate doping modulates the phase in the range from approximately $-180^{\circ}$ to $0^{\circ}$ at frequencies immediately above the resonance frequency in device A [Fig. 3(a)], while simultaneously suppressing the phase in the range from approximately $180^{\circ}$ to $0^{\circ}$ at frequencies slightly below the resonance in device B [Fig. 3(b)]. Consequently, an extremely large phase modulation results within the
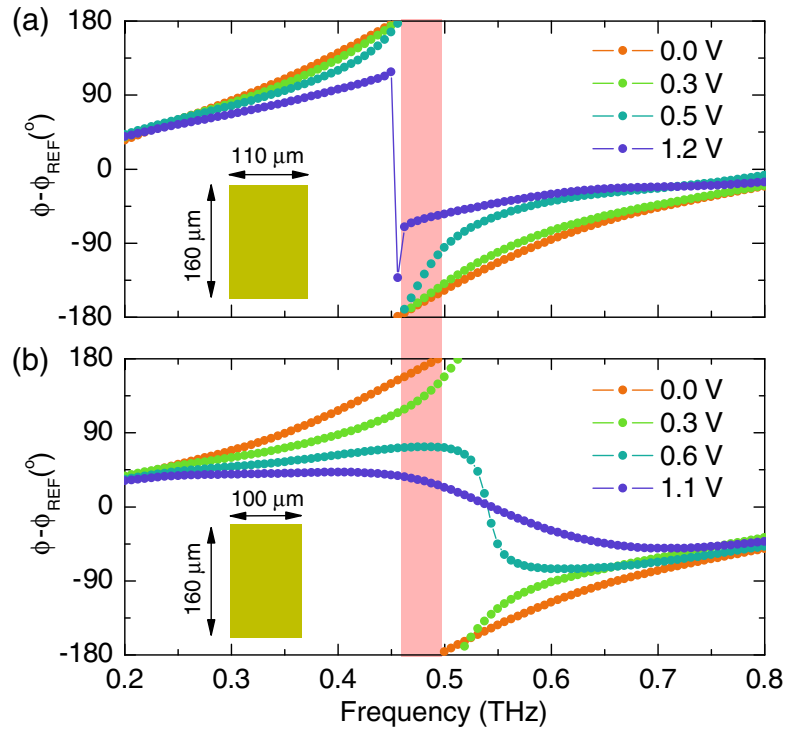

FIG. 3. Full-range gate-controlled phase modulation. Gatedependent reflection phase spectra of (a) device A with a mesa size of $110 \mu \mathrm{m} \times 160 \mu \mathrm{m}$ and (b) device B with a mesa size of $100 \mu \mathrm{m} \times 160 \mu \mathrm{m}$. For both devices, the thickness of the SU8 spacer is $60 \mu \mathrm{m}$, and the mesa array has a period of $240 \mu \mathrm{m}$ in both the $x$ and $y$ directions. The incident THz wave is polarized along the short axis of the mesa. The spectrum measured on an $\mathrm{Al}$ mirror is used as the reference. A large phase modulation is obtained in the shaded region. 
frequency interval between the two resonances [shaded region in Figs. 3(a) and 3(b)], with a maximum modulation range of $243^{\circ}$ at $0.48 \mathrm{THz}$. The range of modulation, although already wide, can be further increased by decreasing the overall absorption of graphene and the SU8 spacer [44]. We note that the wide-range phase modulation is accompanied by a reduction in the reflectance (see Fig. S7 in Supplemental Material [44]), which may limit utilization of the graphene metasurface in applications requiring high light throughput efficiency. Nevertheless, the tunable graphene metasurface may lead to applications where light throughput is not a primary concern. For example, the two metasurfaces may serve as independently gated "phase bits" to actively control the $\mathrm{THz}$ wave fronts; a device design protocol using such phase bits is presented in Appendix D. In addition, our metasurfaces may provide building blocks to realize tunable mathematical operations $[45,46]$, where strong reflectance modulation is needed.

The gate-induced critical transition in the graphene metasurfaces - the main cause of the observed large phase modulation - can be qualitatively understood by modeling the system as a one-port resonator (with resonance frequency $f_{0}$ ) within the framework of CMT [39]. The Al plane completely eliminates the transmission through the resonator, and only the reflection channel must be considered (hence, the name "one port"). The loss in the resonator occurs for two reasons: absorption within the resonator and radiation leakage to external modes. The loss resulting from absorption is denoted as intrinsic $\operatorname{loss} \Gamma_{i}$, whereas that caused by radiation is termed radiation loss and is represented by $\Gamma_{r}$. Analysis based on CMT showed that the reflection coefficient $r$ of such a system can be generally expressed as [39]

$$
r=-1+\frac{2 \Gamma_{r}}{-i\left(f-f_{0}\right)+\Gamma_{i}+\Gamma_{r}}
$$

with $f$ being the incident wave frequency. The evolution of $r$ is most accurately reflected by Smith curves, which are traces of $r$ on the complex plane as $f$ increases from 0 to $\infty$. All of the curves start and end at points close to $r=-1$ [Fig. 2(e)]. However, they cross the real axis (when in resonance, i.e., $f=f_{0}$ ) at locations determined by $r$, where $r=\left(\Gamma_{r}-\Gamma_{i}\right) /\left(\Gamma_{r}+\Gamma_{i}\right)$; the sign of $\Gamma_{r}-\Gamma_{i}$ dictates whether the curves enclose the origin. Thus, the relative amplitudes of $\Gamma_{r}$ and $\Gamma_{i}$ govern the behavior of the reflection phase as $f$ passes the resonance frequency. When $\Gamma_{r}<\Gamma_{i}$, the Smith curve does not enclose the origin; therefore, the resulting phase variation is less than $180^{\circ}$ across the resonance [Fig. 2(e), red curve]. However, a behavioral transition occurs at $\Gamma_{r}>\Gamma_{i}$, and the Smith curve encloses the origin, causing the reflection phase to undergo a full $360^{\circ}$ variation [Fig. 2(e), blue curve]. A transition from an underdamped to an overdamped oscillator occurs at the critical damping $\left(\Gamma_{r}=\Gamma_{i}\right)$ as $\Gamma_{i}$ is increased [see Fig. 2(e)]. Such a transition fully explains the critical transition that we observe in our metasurfaces [Figs. 2(a)-(d), 4(a)-(d)].

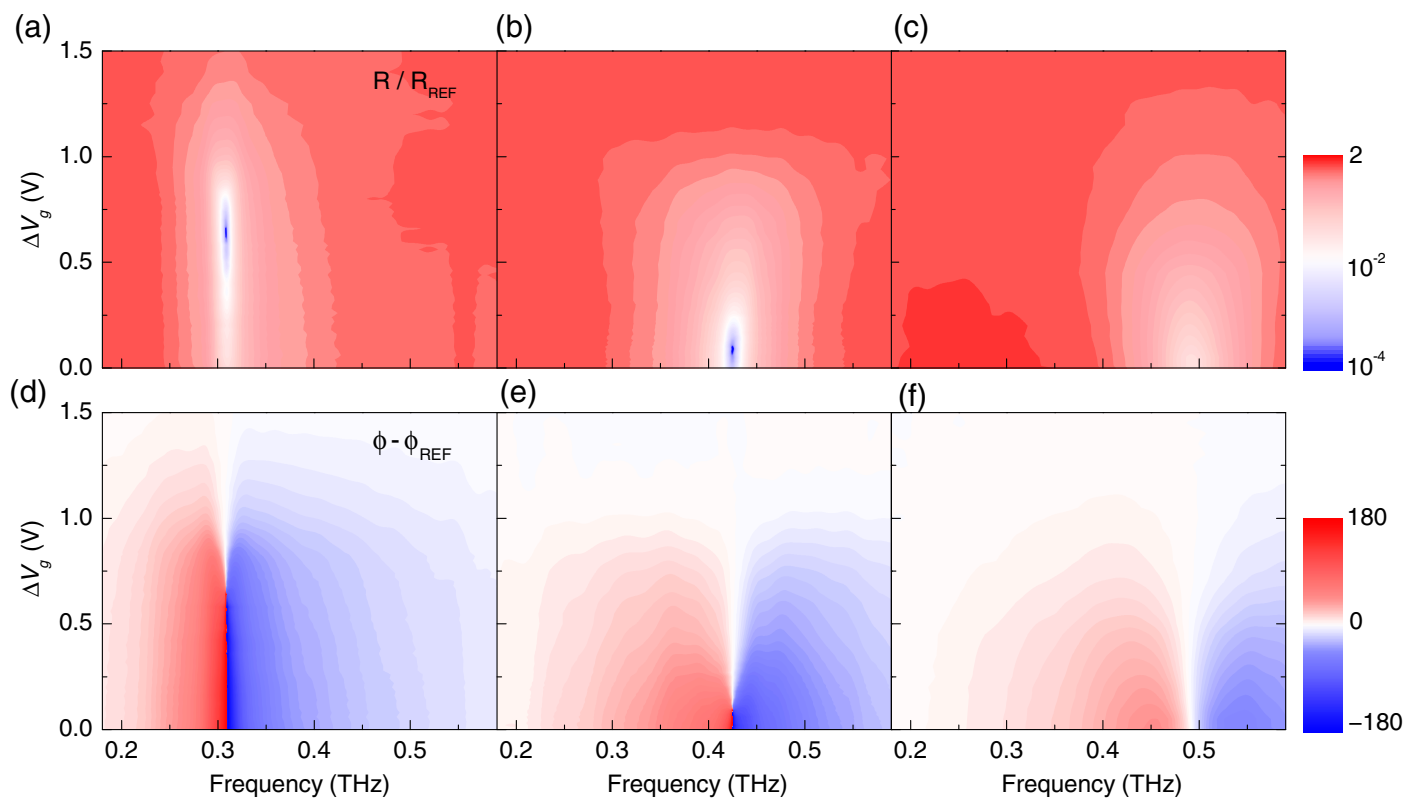

FIG. 4. Reflectance and phase modulation for metasurfaces with different spacer thicknesses. Reflectance modulations measured as a function of both gate voltage and frequency for devices with SU8 spacer thicknesses of (a) $85 \mu \mathrm{m}$, (b) $60 \mu \mathrm{m}$, and (c) $40 \mu \mathrm{m}$. Data from these three devices are normalized to spectra measured at $\Delta V_{g}=2.02 \mathrm{~V}, \Delta V_{g}=2.03 \mathrm{~V}$, and $\Delta V_{g}=1.73 \mathrm{~V}$, respectively. (d)-(f) Reflection phase modulations measured as a function of the gate voltage and frequency for the same three devices considered in (a)-(c). All three devices have a mesa size of $100 \mu \mathrm{m} \times 160 \mu \mathrm{m}$, and the mesa array has a period of $240 \mu \mathrm{m}$ in both the $x$ and $y$ directions. The incident $\mathrm{THz}$ wave is polarized along the long axis of the mesa. 
The proposed resonator model is supported by finitedifference time-domain (FDTD) simulations performed on realistic structures. The simulations quantitatively reproduce all the experimental observations in Figs. 2 and 4 (see also Appendix A). They further show the crucial role played by graphene in driving the critical transition: The doping of graphene essentially increases $\Gamma_{i}$ of our device, but has limited influence on $\Gamma_{r}$, which is predominantly determined by the geometry of the metasurface (see Appendix B).

Valuable insights into the working mechanism of the proposed gate-tunable metasurface can be gained through FDTD simulations. For low graphene doping $\left(\Gamma_{i}<\Gamma_{r}\right)$, the metasurface (including graphene) is highly transparent. The simulations show that $\mathrm{THz}$ waves penetrate deep into the resonator before they are absorbed, and the near-field coupling between the $\mathrm{Al}$ mesa and the $\mathrm{Al}$ plane leads to magnetic resonance. However, at high doping levels $\left(\Gamma_{i}>\Gamma_{r}\right)$, the large size of the absorption prevents the waves from reaching the $\mathrm{Al}$ plane to establish near-field coupling between the mesa and the plane (see Appendix A). The gate-tunable metasurface thus behaves as an electric reflector with a small phase variation across the resonance. Thus, graphene, under gate control, clearly serves as a tunable lossy medium; the variable loss breaks the delicate balance between $\Gamma_{i}$ and $\Gamma_{r}$, thereby inducing a critical transition accompanied by a large phase modulation.

Because $\Gamma_{r}$, and to a large extent $\Gamma_{i}$, depends critically on the metasurface geometry, our model further predicts that the critical transition can also be tuned by modifying the structure of the metasurface. FDTD simulations show that a thinner spacer layer between two metallic layers leads to stronger near-field coupling, resulting in the $Q$ factor of the resonance being larger (and therefore a lower $\Gamma_{r}$ ). A lower $\Gamma_{i}$ is then required at the critical transition; therefore, decreasing the spacer layer thickness shifts the transition point toward low doping levels in graphene. A further decrease in the thickness eventually makes $\Gamma_{r}$ lower than $\Gamma_{i}$, even without contribution from the graphene. Doping graphene then causes only the metasurface to become more overdamped because doping can increase only $\Gamma_{i}$. Consequently, the critical transition point completely disappears. These predictions are finally compared against the gate-frequency phase diagrams obtained for metasurfaces with 60- $\mu \mathrm{m}$ [Figs. 4(b) and 4(e)] and 40- $\mu \mathrm{m}$ [Figs. 4(c) and 4(f)] spacer layers, and the qualitative agreement provides additional support for the proposed model and the involved simulations (see Appendix B).

Having understood the working mechanism of the proposed metasurface, we proceed to formulate a general strategy for designing tunable metastructures with a large phase modulation. We start by analyzing the nature of the critical transition point, which is a precondition for the large phase modulation. At the critical point, $\Gamma_{r}=\Gamma_{i}$ implies that the system acts as a perfect absorber $[47,48]$. For two-port resonators adopted in previous studies [37], the time-reversed version of such a perfect absorber emits symmetrically to both ports of the resonator; therefore, the critical point (perfect absorption) is reached only when the resonator is excited from both ports simultaneously. In a realistic device in which the resonator is excited from only one side, the critical condition can never be satisfied for finite $\Gamma_{i}$. The lack of a critical transition is shown clearly in the Smith curves of the transmission coefficient $t$ for the two-port model [Fig. 2(f)]. For this model, all the curves do not enclose the origin, even in the limiting case $\Gamma_{i}=0$ [Fig. 2(f), broken line], and lead to a limited phase modulation, a problem that hindered previous attempts [37]. Analyzing these competing schemes provides a general guideline for designing graphene-based phase modulators: use systems that support the critical point and use graphene as a tunable loss material to access both sides of the critical transition. Detailed discussions are presented in Appendix C.

Finally, we demonstrate a gate-tunable polarizer using the proposed graphene metasurface [Fig. 5(e)]. The Al mesas are replaced by stripes; thus, the system exhibits magnetic resonance only for polarization in the $x$ direction $(\vec{E} \| \hat{x})$. On being gated, graphene significantly modulates both the reflectance and phase spectra [Figs. 5(a) and 5(b)] because of the critical transition accompanied by a large phase modulation. However, the reflection for waves polarized in the $y$ direction is hardly tuned by graphene because of the lack of resonance [44]. Such an anisotropic response can be readily used for controlling the polarization. As shown in Figs. 5(c) and 5(d), the reflectance ratio $\left|r_{x}\right| /\left|r_{y}\right|$ and phase difference $\phi_{x}-\phi_{y}$ in the $x$ and $y$ directions are effectively tuned by varying $V_{g}$, leading to a dramatic modulation in the polarization state of the reflected wave. As an example, we fix the frequency at $0.63 \mathrm{THz}$ and linearly polarize the incident wave at an angle of $32^{\circ}$ with respect to the $x$ axes. The reflected wave is thereby elliptically polarized because of the anisotropic response of our polarizer. The polarization of the reflected wave, characterized by two parameters [the ratio between the semiminor and semimajor axes of the ellipse $(S / L)$, and the angle $\theta$ between the semi-major and $x$ axis; inset of Fig. 5(e)], could then be widely tuned using graphene under gate control [Fig. 5(f)]. The working efficiency of our tunable polarizer is $\sim 40 \%$, as a result of the reflectance reduction for $x$ polarization [44].

In conclusion, we propose a novel loss-driven mechanism to achieve a wide-range phase modulation and experimentally demonstrate the mechanism in the THz regime with metasurfaces integrating magnetic resonators and gate-controlled graphene. The overall thicknesses of the metasurfaces are in the deep subwavelength regime. A one-port resonator model explains the essential features of the proposed metasurfaces, and further reveals the crucial role of graphene as a gatetunable loss in modulating the critical transition in the resonator, leading to an extremely large phase modulation. 
(a)

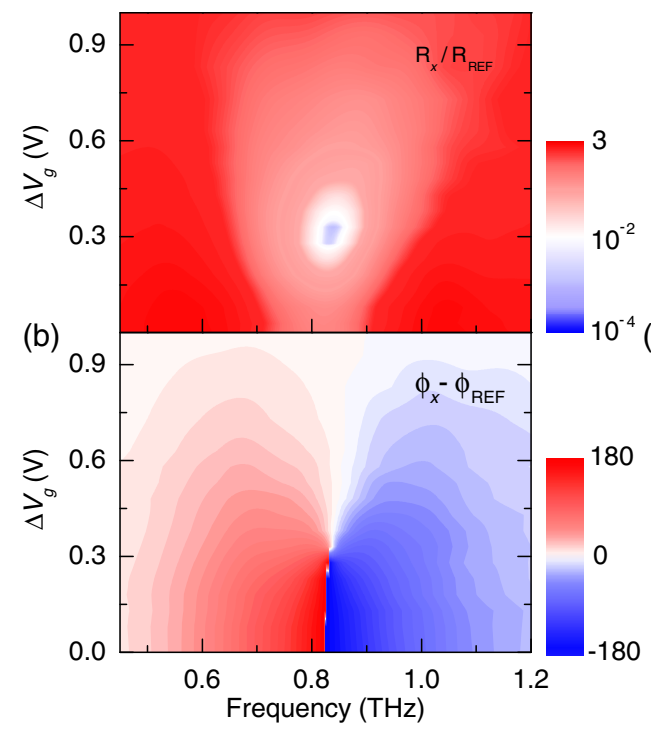

(c)

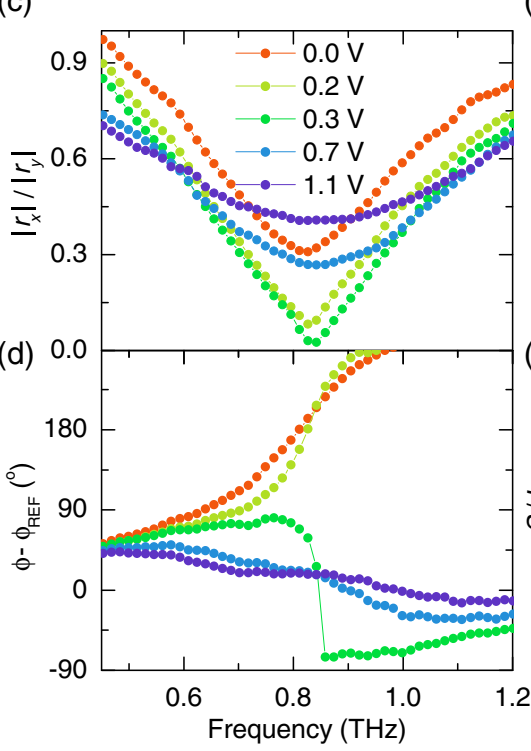

(e)

FIG. 5. A gate-tunable THz polarizer. (a) A reflectance modulation and (b) reflection phase modulation as a function of the gate voltage and frequency measured on the polarizer [shown in (e)]. The incident THz wave is polarized perpendicular to the stripes of the polarizer. (c) The reflection amplitude ratio and (d) phase difference between waves with polarizations in the $x$ and $y$ directions measured as a function of the frequency at different gate voltages. Both parameters are effectively modulated by the gate voltage. (e) An optical image of the polarizer. The stripes have a width of $60 \mu \mathrm{m}$, and they are spaced $60 \mu \mathrm{m}$ apart. (f) The polarization state (see inset) of the reflected $\mathrm{THz}$ wave, characterized by the elliptical axis ratio $(S / L$, red curve) and the rotation angle $\theta$ (blue curve), measured as a function of the gate voltage. The working frequency is $0.63 \mathrm{THz}$. The gate causes a drastic modulation of the polarization state.

Our full-range tunable local phase control opens the door to exciting photonic applications in the THz regime. We present a gate-tunable polarizer as an early demonstration of the capability of our graphene metasurfaces.

We thank Feng Wang, Meng Qiu, and Shulin Sun for helpful discussions. Part of the sample fabrication was performed at Fudan Nano-fabrication Lab. Z. M., X. L., Q. H., K.D., and L.Z. were supported by the National Natural Science Foundation (NSF) of China under Grants No. 11474057, No. 11174055, and No. 11204040. Q. W. and Y.Z. acknowledge financial support from the National Basic Research Program of China (973 Program) under Grants No. 2011CB921802 and No. 2013CB921902. Z. M. performed the calculations and simulations and designed the metasurfaces. Q. W. and X. L. fabricated the samples. Z. M., Q. W., X. L., and Q.H. performed the THz measurements. K. D. assisted with the simulations. Z. A. helped with sample fabrications. L. Z. and Y. Z. initiated, designed, and co-supervised the project. Z. M., L. Z., and Y.Z. wrote the manuscript and all authors commented on it.

Z. M., Q. W., and X. L. equally contributed to this work.

\section{APPENDIX A: FDTD SIMULATIONS OF THE PROPOSED METASURFACES}

\section{Simulation model}

The conductivity of graphene in the $\mathrm{THz}$ regime can be described by the linear-response theory under the random phase approximation, which comprises only intraband contributions [49]:

$$
\sigma(\omega) \approx \frac{i e^{2} v_{f} \sqrt{n}}{\hbar \sqrt{\pi}(\omega+i \Gamma)},
$$

where $v_{f}$ is the Fermi velocity of electrons in graphene (approximately $9 \times 10^{5} \mathrm{~m} / \mathrm{s}$ ) [24], $n$ is the two-dimensional electron density of graphene, and $\Gamma$ is a phenomenological constant accounting for the electron scattering rate (damping). In the FDTD simulations, graphene was modeled as a thin dielectric layer of thickness $d$ and with an anisotropic dielectric function [50] given by $\stackrel{\leftrightarrow}{\varepsilon}_{r}=\operatorname{diag}\left[\varepsilon_{r}^{\|}, \varepsilon_{r}^{\|}, 1\right]$, where

$$
\varepsilon_{r}^{\|}=\sqrt{\varepsilon_{r 1} \varepsilon_{r 2}}-\frac{\sigma(\omega)}{i \varepsilon_{0} \omega d}
$$

is the standard Drude form. Here, $\varepsilon_{r 1}$ and $\varepsilon_{r 2}$ are dielectric constants of two media surrounding graphene and $d$ is set as $1 \mathrm{~nm}$. Notably, $d$ is much larger than the real thickness of graphene, $0.3 \mathrm{~nm}$. However, it was shown in Ref. [37] that the exact value of $d$ does not influence the final result as long as $d$ is considerably smaller than the wavelength, which is the case in our simulations. We assume that $n$ is related to the gate voltage $\Delta V_{g}$ through $n=\left(n_{0}^{2}+\alpha^{2}\left|\Delta V_{g}\right|^{2}\right)^{1 / 2}$, where $\alpha$ is the gate capacitance in the ion-gel-based gating scheme and $n_{0}$ is 


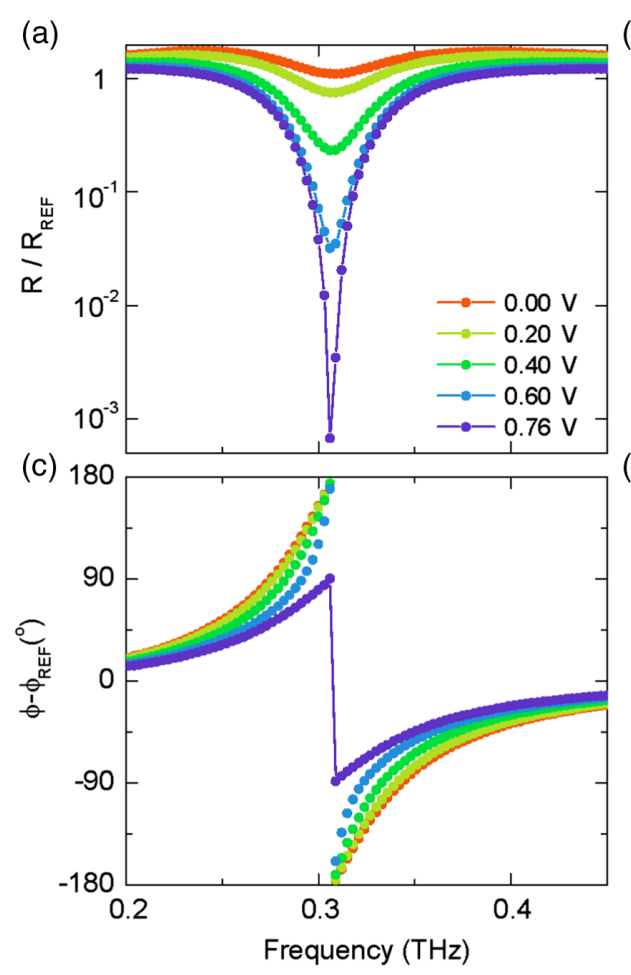

(b)

(d)
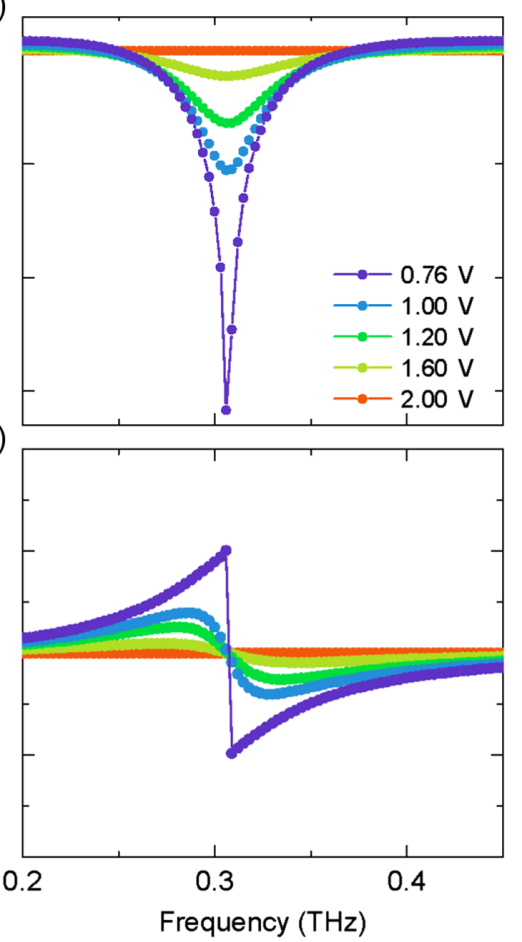

FIG. 6. FDFD simulations for the experiments described in Fig. 2. (a),(b) Variation of the reflectance $\left(R=|r|^{2}\right)$ with the relative gate voltage $\Delta V_{g}$. A critical transition occurs at $\Delta V_{c}=0.76 \mathrm{~V}$. The reflectances for $\Delta V_{g} \leq \Delta V_{c}$ and $\Delta V_{g} \geq \Delta V_{c}$ are shown in (a) and (b), respectively. The spectrum calculated at $\Delta V_{g}=2.02 \mathrm{~V}$ is used as the reference. (c),(d) Phase modulation for $\Delta V_{g} \leq \Delta V_{c}$ and $\Delta V_{g} \geq \Delta V_{c}$, respectively. The simulated system has geometrical parameters identical to those of the system studied in Fig. 2 .

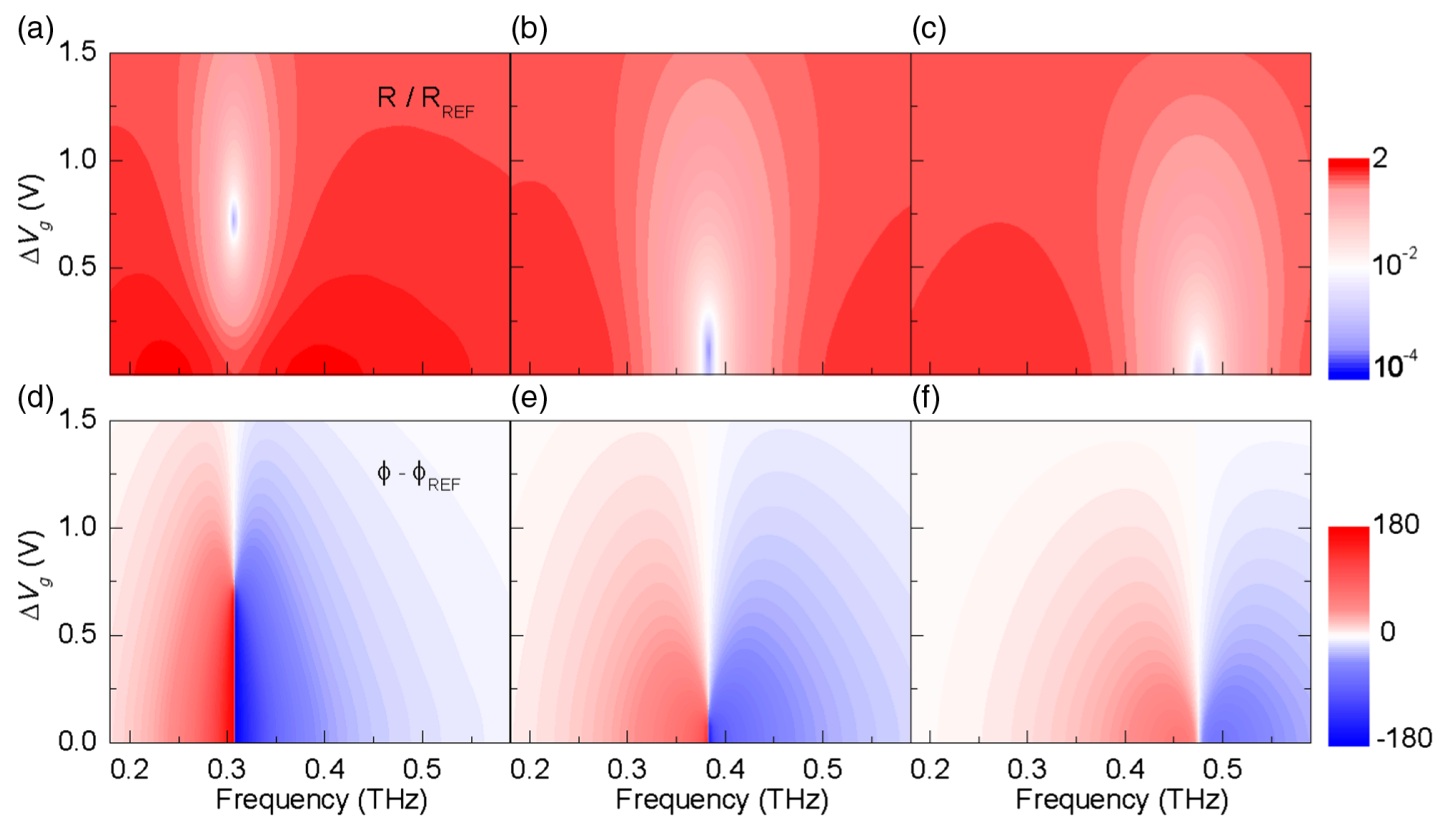

FIG. 7. FDTD simulations for the experiments described in Figs. 4. (a)-(c) Reflectance modulation as a function of both gate voltage and frequency for the devices with SU8 spacer thicknesses of 85, 60, and $40 \mu \mathrm{m}$, respectively. Data from these three devices are normalized by the spectra calculated at $\Delta V_{g}$ values of 2.02,2.03, and $1.73 \mathrm{~V}$, respectively. (d)-(f) Reflection phase modulation as a function of the gate voltage and frequency for the same devices in (a)-(c). The simulated devices have geometrical parameters identical to those of the devices shown in Fig. 4. 
the residual carrier density [37], determined by fitting experimental data.

We treat $\mathrm{Al}$ as a lossy metal in the FDTD simulations. The optimal simulation results are obtained when the electrical conductivity of $\mathrm{Al}$ is set to $10^{6} \mathrm{~S} / \mathrm{m}$, which is considerably lower than the bulk value of $3.5 \times 10^{7} \mathrm{~S} / \mathrm{m}$ [51]. Such a difference in the electrical conductivity between bulk and thin-film $\mathrm{Al}$ has also been observed in previous $\mathrm{THz}$ measurements [52]. The SU8 spacer is treated as a dielectric insulator with $\operatorname{Re}(\varepsilon)=3.5$ and $\operatorname{Im}(\varepsilon)=0.28$. All of these parameters are determined by carefully comparing the FDTD results with experimental data for various samples (metasurfaces without graphene).

\section{Simulation results and discussions}

We perform extensive FDTD simulations for all of the cases experimentally characterized in the main text. Figures 6 and 7 show the FDTD results for the cases presented in Figs. 2 and 4, respectively. In the simulations, the optimal results are obtained for the following parameters: $n_{0}=9.04 \times 10^{11} \mathrm{~cm}^{-2}$ and $\alpha=3.35 \times 10^{12} \mathrm{~cm}^{-2} \mathrm{~V}^{-1}$ for the $85-\mu \mathrm{m}$ case, $n_{0}=2.34 \times 10^{12} \mathrm{~cm}^{-2}$ and $\alpha=$ $5.89 \times 10^{12} \mathrm{~cm}^{-2} \mathrm{~V}^{-1}$ for the $60-\mu \mathrm{m}$ case, and $n_{0}=$ $2.31 \times 10^{12} \mathrm{~cm}^{-2}$ and $\alpha=6.50 \times 10^{11} \mathrm{~cm}^{-2} \mathrm{~V}^{-1}$ for the $40-\mu \mathrm{m}$ case. We attribute the different values to the different local environments of graphene in the different samples. The simulations reproduce all essential features, particularly the experimentally observed critical transitions tuned by the gate and SU8 spacer thickness.

To acquire a deeper understanding of the resonance in the underdamped and overdamped regimes, we perform FDTD simulations to calculate the field patterns for two representative cases. The field patterns for the underdamped and overdamped metasurfaces are shown in Figs. 8(a) and 8(b), respectively. In the case of the underdamped resonator [Fig. 8(a)], waves penetrate deep into the metasurface, establishing near-field coupling between the two metallic layers, and causing magnetic resonance. By contrast, waves are directly reflected by the top mesa layer, preventing them from entering the metasurface [Fig. 8(b)]. This explains the simulated metasurface as an electric reflector when it is located in the overdamped region; specifically, only the top mesa layer reflects $\mathrm{THz}$ waves.

\section{APPENDIX B: GRAPHENE AS A TUNABLE LOSS IN THE METASURFACE: ANALYSIS BASED ON CMT}

To understand the crucial role of graphene in modulating the resonance behavior of the metasurfaces, we analyze the effect of graphene doping by using the CMT formula in Eq. (1). Although in principle the CMT is quantitatively valid only for high- $Q$ resonators [39], our analyses (presented below) show that the qualitative behavior of the resonant systems (with moderate $Q$ factors) is effectively described by the CMT.

We determine the radiation loss $\Gamma_{r}$ and intrinsic loss $\Gamma_{i}$ at different gate voltages by fitting the corresponding FDTD simulation results with Eq. (1). We choose to fit the FDTD simulation results rather than experimental data because (a) all experimental features are effectively reproduced by the FDTD simulations and (b) the fitting is highly sensitive to the shape of the curve at the resonance; fluctuations in the measured spectra render any fitting to experimental data unreliable. Because the CMT is valid only at frequencies near the resonance [39], to obtain reliable fitting results, we perform the fitting procedure in a frequency interval

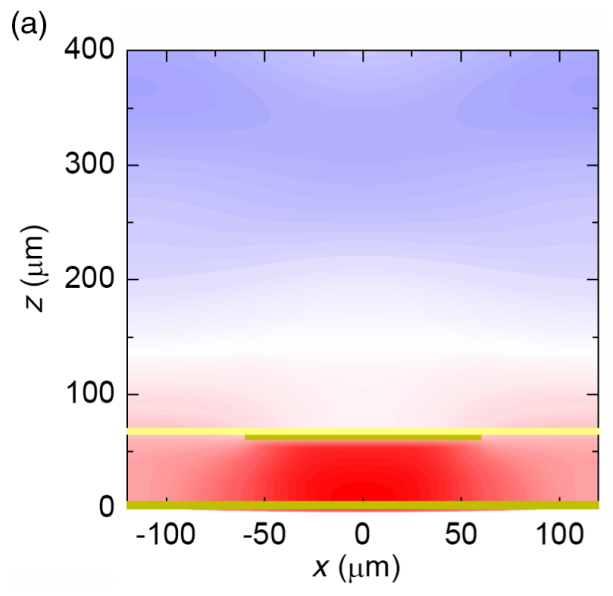

(b)

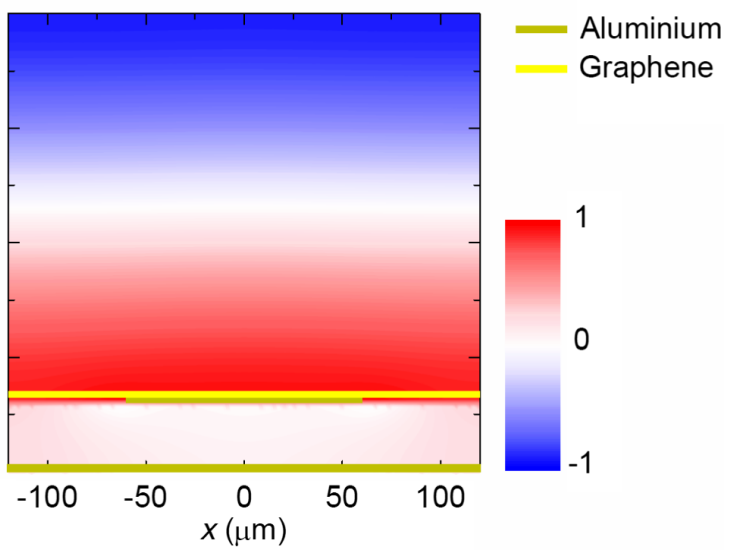

FIG. 8. Comparison of the field pattern between the underdamped and overdamped resonators. (a) Simulated $H_{y}$ distribution in the $x z$ plane for the graphene metasurface with a 60- $\mu \mathrm{m}$-thick SU8 spacer layer. The size of the Al mesa is $160 \mu \mathrm{m} \times 120 \mu \mathrm{m}$, and the mesa array periodicity is fixed at $240 \mu \mathrm{m} \times 240 \mu \mathrm{m}$ (only one mesa is shown here). The carrier density of graphene is set at $2.0 \times 10^{11} \mathrm{~cm}^{-2}$, which places the resonator in the underdamped region. (b) Simulated field distribution when the resonator is in the overdamped region (graphene carrier density $1.45 \times 10^{13} \mathrm{~cm}^{-2}$ ). In both cases, the devices are illuminated by normally incident plane waves polarized such that $\vec{E} \| \vec{x}$. 

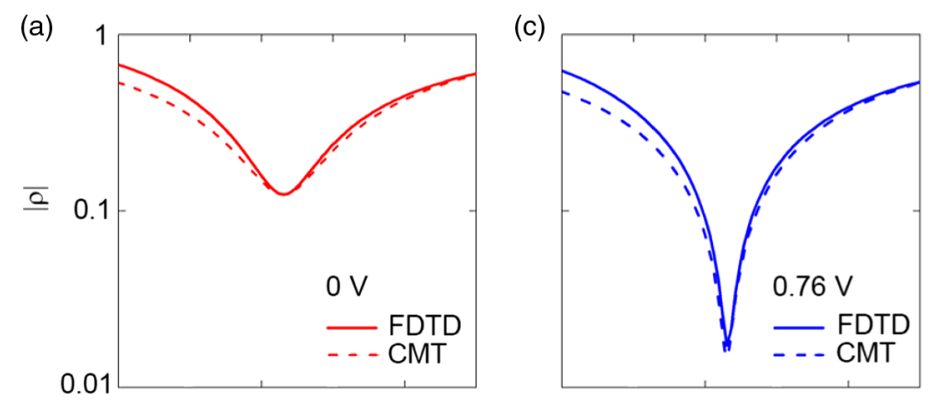

(e)
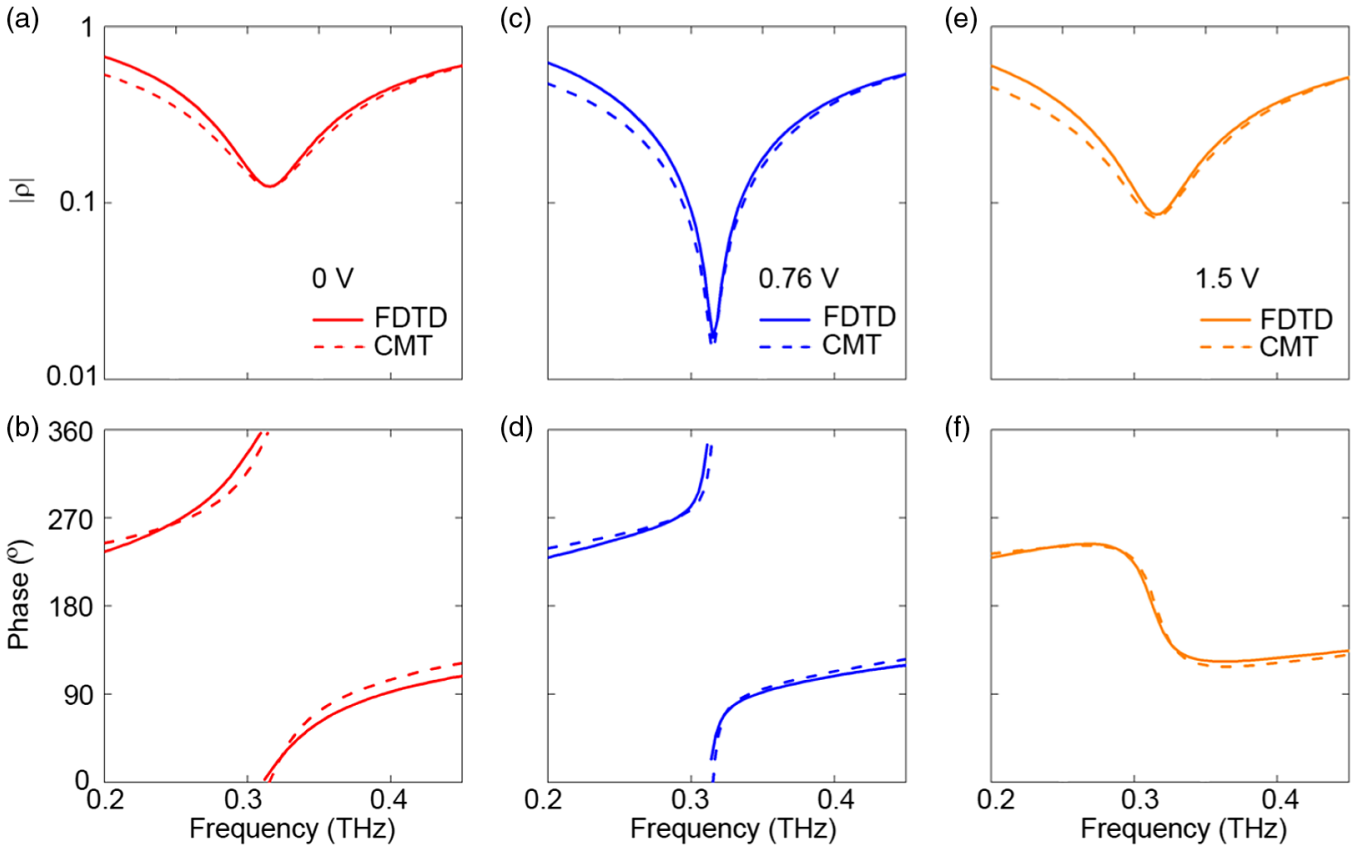

FIG. 9. Reflection spectra calculated from FDTD and CMT results for the $85-\mu \mathrm{m}$-thick spacer metasurface for different gating voltages. The CMT parameters $\Gamma_{i}$ and $\Gamma_{r}$ differ among the panels. (a),(b) $\Gamma_{i}=0.285 f_{0}$ and $\Gamma_{r}=0.223 f_{0},(\mathrm{c}),(\mathrm{d}) \Gamma_{i}=0.281 f_{0}$ and $\Gamma_{i}=0.281 f_{0}$, (e),(f) $\Gamma_{i}=0.287 f_{0}$ and $\Gamma_{r}=0.370 f_{0}$.

centered at the resonance. We then vary the bandwidth of the interval and ensure that the obtained fitting results converge and are nearly independent of the bandwidth. Figure 9 shows a comparison of the FDTD-simulated and CMT-fitted results for the metasurface with an $85-\mu \mathrm{m}$-thick spacer for three typical gating voltages. Reasonable agreement between the two results can be seen. In particular, the qualitative behavior of the resonance (i.e., at a phase variation of $360^{\circ}$ or less than $180^{\circ}$ ) is effectively reproduced by the CMT results.

Figure 10 shows how the obtained values of $\Gamma_{r}$ and $\Gamma_{i}$, scaled by the corresponding resonance frequency $f_{0}$, vary with the gate voltage $\Delta V_{g}$ in different devices. Two conclusions can be drawn from this result. First, increasing the carrier density in graphene (by increasing $\Delta V_{g}$ ) increases $\Gamma_{i}$ but leaves $\Gamma_{r}$ almost unchanged. Second, when the graphene doping level is fixed, the metasurface with the thinner spacer layer exhibits lower radiation loss. Therefore, sections of the resonances in the metasurfaces with $85-$ and $60-\mu \mathrm{m}$ spacer layers are located in the underdamped region, whereas all resonances in the metasurface with a $40-\mu \mathrm{m}$-thick spacer layer are located in the overdamped region because of lower radiation loss of the system. The device with a 40- $\mu \mathrm{m}$-thick spacer does not show a transition with an increase in $\Delta V_{g}$ because gating can only increase the intrinsic loss, driving the system farther away from the transition boundary.

Figure 10 clearly shows that $\Gamma_{r}$ varies with the spacer layer thickness. We perform FDTD simulations to further quantify the effect of the spacer layer thickness on $\Gamma_{r}$.
We compute the $Q$ factor (which is inversely proportional to $\Gamma_{r}$ ) of a series of devices with different spacer thicknesses. To specifically delineate the effect of the spacer thickness on the radiation loss, all materials (both metal and SU8) are assumed to be dissipationless (i.e., the intrinsic loss is set to 0 ). In Fig. 11, the $Q$ factor is shown as a

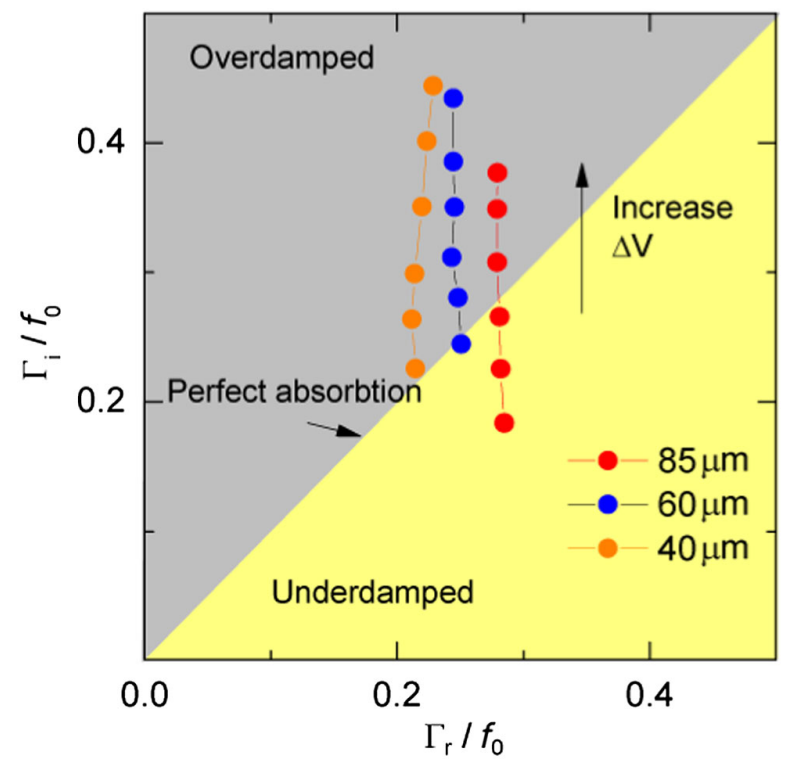

FIG. 10. Critical transition modulated by doping and the spacer layer thickness. We determine $\Gamma_{r}$ and $\Gamma_{i}$ by fitting FDTD simulation results with Eq. (1). Three device structures (with spacer layer thicknesses 40,60, and $85 \mu \mathrm{m}$ ) are examined, and the relative gate voltage is varied from 0 to $1.5 \mathrm{~V}$. 


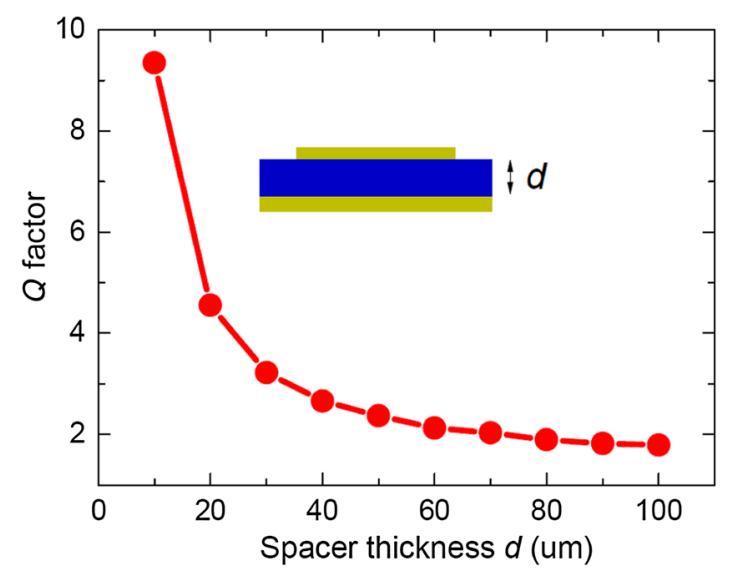

FIG. 11. $Q$ factors as a function of the spacer layer thickness. All materials in the metasurface are assumed to be dissipationless. The geometry of the $\mathrm{Al}$ mesa layer is identical to the geometry shown in Fig. 4, and only $d$ is varied.

function of the spacer layer thickness. A thinner spacer layer corresponded to a higher $Q$ factor, which explains the decrease in the radiation loss with a decrease in the spacer layer thickness (Fig. 10). A metasurface with a thinner spacer shows a stronger near-field coupling between two metallic layers, leading to a higher $Q$ factor and therefore lower $\Gamma_{r}$. Such an intriguing effect has previously been discussed for a similar system [53].

\section{APPENDIX C: CMT-BASED ANALYSIS OF A TWO-PORT RESONATOR}

According to the CMT [39], for a background medium (without the resonant structure) that is completely transparent (i.e., $t_{0}=1, r_{0}=0$ ), we obtain the following expressions for the two-port, single-mode model:

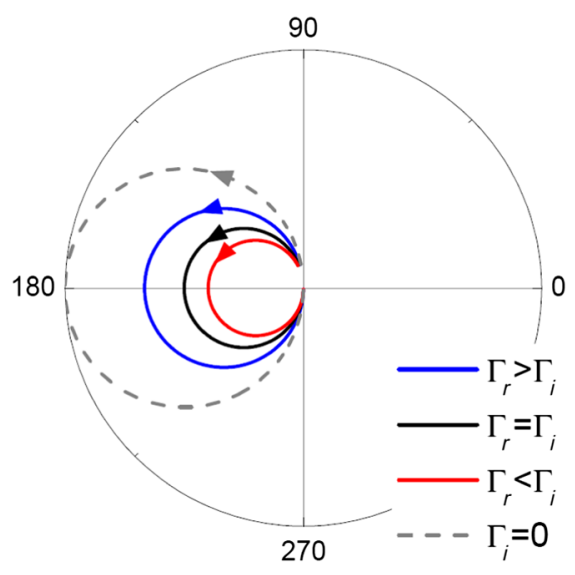

FIG. 12. CMT analysis of a two-port, single-mode resonator. Smith curves for $r$ according to Eq. (C1). Four representative cases are plotted: $\Gamma_{i}=0.05 f_{0}$ and $\Gamma_{r}=0.1 f_{0}$ (blue curve), $\Gamma_{i}=0.1 f_{0}$ and $\Gamma_{r}=0.1 f_{0}$ (black curve), $\Gamma_{i}=0.15 f_{0}$ and $\Gamma_{i}=0.15 f_{0}, \Gamma_{r}=0.1 f_{0}$ (red curve), $\Gamma_{i}=0$ and $\Gamma_{r}=0.1 f_{0}$ (gray curve).
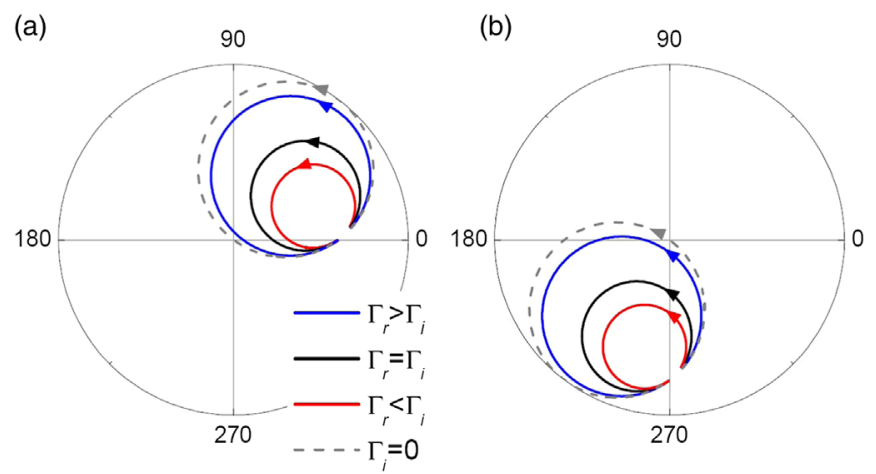

FIG. 13. CMT analysis of a two-port, single-mode resonator. (a),(b) Smith curves for $r$ and $\mathrm{t}$ according to Eq. (C2), respectively. Four representative cases are plotted: $\Gamma_{i}=0.05 f_{0}$ and $\Gamma_{r}=0.1 f_{0}$ (blue curve), $\Gamma_{i}=0.1 f_{0}$ and $\Gamma_{r}=0.1 f_{0}$ (black curve), $\Gamma_{i}=0.15 f_{0}$ and $\Gamma_{r}=0.1 f_{0}$ (red curve), and $\Gamma_{i}=0$ and $\Gamma_{r}=0.1 f_{0}$ (gray curve). Without loss of generality, the reflection and transmission coefficients of the background medium are set as $r_{0}=0.6$ and $t_{0}=-0.8 i$.

$$
r=\frac{\Gamma_{r}}{i\left(f-f_{0}\right)-\Gamma_{i}-\Gamma_{r}}, \quad t=1+\frac{\Gamma_{r}}{i\left(f-f_{0}\right)-\Gamma_{i}-\Gamma_{r}} .
$$

The Smith curves for the transmission coefficient $t$ are plotted in Fig. 2(f). Here, we present the Smith curves for the reflection coefficient $r$ in Fig. 12. Again, no critical transition is observed in $r$ when the doping level is varied.

In general, the background medium may not be completely transparent, but it shows finite transmission $\left(t_{0}\right)$ and reflection $\left(r_{0}\right)$. A generalized model based on the CMT yields

$$
\begin{aligned}
& r=r_{0}+\frac{\Gamma_{r} e^{i \Phi}}{i\left(f-f_{0}\right)-\Gamma_{i}-\Gamma_{r}}, \\
& t=t_{0}+\frac{\Gamma_{r} e^{i \Phi}}{i\left(f-f_{0}\right)-\Gamma_{i}-\Gamma_{r}},
\end{aligned}
$$

with $e^{i \Phi}=-\left(t_{0}+r_{0}\right) /\left|t_{0}+r_{0}\right|$ [39]. Calculations based on Eq. (C2) show that the Smith curves are now tilted (Fig. 13). However, our conclusion (i.e., lack of critical transition in two-port resonators) still holds, even in the limiting case $\Gamma_{i}=0$ (broken lines, Fig. 13).

\section{APPENDIX D: GATE-TUNABLE GRATING BASED ON GRAPHENE METASURFACES}

In the main text, we demonstrate the active modulation of the reflection phase by almost $2 \pi$ by using two independently gated graphene metasurfaces (Fig. 3). Here, we show how these two metasystems can be used as basic "phase bits" to actively control the diffraction of $\mathrm{THz}$ waves. Specifically, we demonstrate a gate-tunable 


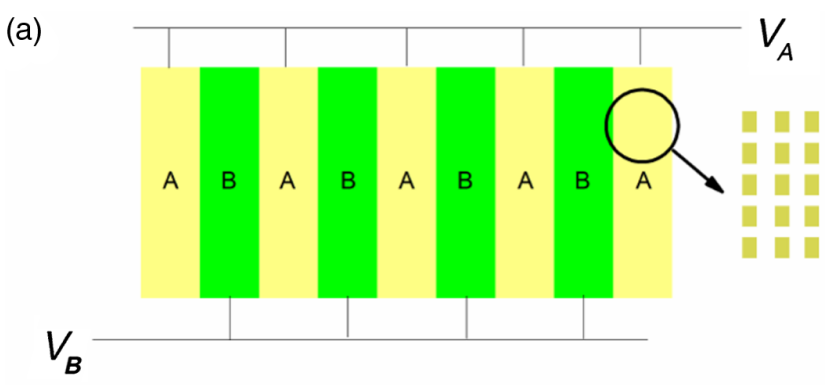

(b)

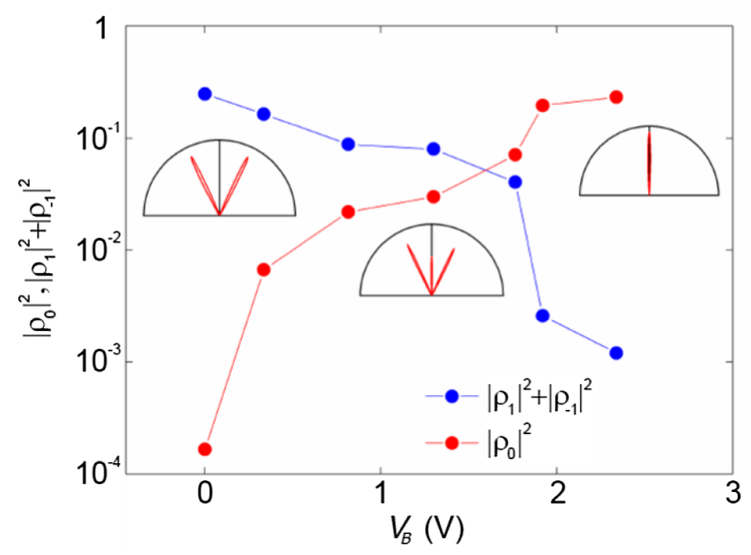

FIG. 14. Numerical simulation of a gate-tunable diffraction grating. (a) Schematics of the device with the stripes A and B, consisting of pixels of the same size as that of the pixels in samples A and B in Fig. 3. (b) Plots of the zero- and first-order reflectances of the device against $V_{B}$ for $V_{A}=1.32 \mathrm{~V}$ and $f=0.48 \mathrm{THz}$. The insets depict the normalized scattering patterns of the device for normally incident plane waves at three typical gating voltages $\left(V_{B}=0,1.29\right.$, and $\left.2.34 \mathrm{~V}\right)$.

diffraction grating by using numerical simulations (COMSOL Multiphysics).

The device configuration is shown in Fig. 14(a). The metasurface consists of two types of stripes [stripes $A$ and B, shown in Fig. 14(a)] ordered in a one-dimensional AB lattice. Stripes A and B are $720-\mu \mathrm{m}$ wide and consist of pixels of the same size as those in samples A and B discussed in Fig. 3 [inset of Fig. 14(a)]. Two independent external gates control the doping of stripes $\mathrm{A}$ and $\mathrm{B}$ [Fig. 14(a)]. The simulation shows that the reflection wave front of the device could be modulated by varying the two gate voltages $V_{A}$ and $V_{B}$. Figure 14(b) shows how the zeroorder reflectance (denoted by $\left|\rho_{0}\right|^{2}$ ) and the first-order reflectance (denoted by $\left|\rho_{ \pm 1}\right|^{2}$ ) vary with $V_{B}$ when $V_{A}$ is fixed at $1.32 \mathrm{~V}$ and $f=0.48 \mathrm{THz}$. The device behaves as a grating (with predominantly first-order diffractions) at $V_{B}=0 \mathrm{~V}$, and it acts as a normal mirror (with only specular reflection) when $V_{B}$ increases to $2.34 \mathrm{~V}$ [insets of Fig. 14(b)].

The mechanism of such a gate-tunable diffraction gating is as follows: $V_{B}$ continuously modulates the phase difference $\left(\phi_{A}-\phi_{B}\right)$ between the waves reflected by the two stripes. The phases cancel each other at $V_{B}=0 \mathrm{~V}$ (where $\phi_{A}-\phi_{B} \approx 180^{\circ}$ ), leaving only first-order diffractions; however, the phases reinforce each other at $V_{B}=2.34 \mathrm{~V}$ (where $\phi_{A}-\phi_{B} \approx 0^{\circ}$ ), resulting in the domination of specular reflection.

In the simulation, we choose gating voltages such that the reflection amplitudes of the stripes $\mathrm{A}$ and $\mathrm{B}$ are nearly identical in the two extreme cases (i.e., $V_{B}=0,2.34 \mathrm{~V}$ ). Two parameters are fixed: $\alpha=2 \times 10^{13} \mathrm{~cm}^{-2} \mathrm{~V}^{-1}$ and $n_{0}=2.26 \times 10^{11} \mathrm{~cm}^{-2}$. Without loss of accuracy, we simplify the simulation by replacing the stripes with their effective media that have the same reflection amplitudes and phases.

[1] M. Born and E. Wolf, Principles of Optics: Electromagnetic Theory of Propagation, Interference and Diffraction of Light (Cambridge University Press, Cambridge, England, 1999).

[2] S. Larouche, Y.-J. Tsai, T. Tyler, N. M. Jokerst, and D. R. Smith, Infrared Metamaterial Phase Holograms, Nat. Mater. 11, 450 (2012).

[3] X. Ni, A. V. Kildishev, and V. M. Shalaev, Metasurface Holograms for Visible Light, Nat. Commun. 4, 2807 (2013).

[4] W. T. Chen et al., High-Efficiency Broadband MetaHologram with Polarization-Controlled Dual Images, Nano Lett. 14, 225 (2014).

[5] Y. Yifat, M. Eitan, Z. Iluz, Y. Hanein, A. Boag, and J. Scheuer, Highly Efficient and Broadband Wide-Angle Holography Using Patch-Dipole Nanoantenna Reflectarrays, Nano Lett. 14, 2485 (2014).

[6] P. Genevet and F. Capasso, Holographic Optical Metasurfaces: A Review of Current Progress, Rep. Prog. Phys. 78, 024401 (2015).

[7] J. Hao, Y. Yuan, L. Ran, T. Jiang, J. A. Kong, C. T. Chan, and L. Zhou, Manipulating Electromagnetic Wave Polarizations by Anisotropic Metamaterials, Phys. Rev. Lett. 99, 063908 (2007).

[8] A. Pors, M. G. Nielsen, R. L. Eriksen, and S. I. Bozhevolnyi, Broadband Focusing Flat Mirrors Based on Plasmonic Gradient Metasurfaces, Nano Lett. 13, 829 (2013).

[9] N. Yu, P. Genevet, M. A. Kats, F. Aieta, J.-P. Tetienne, F. Capasso, and Z. Gaburro, Light Propagation with Phase Discontinuities: Generalized Laws of Reflection and Refraction, Science 334, 333 (2011).

[10] A. S. Roberts, A. Pors, O. Albrektsen, and S. I. Bozhevolnyi, Subwavelength Plasmonic Color Printing Protected for Ambient Use, Nano Lett. 14, 783 (2014).

[11] Y. Jin, H. Tai, A. Hiltner, E. Baer, and J. S. Shirk, New Class of Bioinspired Lenses with a Gradient Refractive Index, J. Appl. Polym. Sci. 103, 1834 (2007).

[12] J. B. Pendry, A. J. Holden, D. J. Robbins, and W. J. Stewart, Magnetism from Conductors and Enhanced Nonlinear Phenomena, IEEE Trans. Microwave Theory Tech. 47, 2075 (1999).

[13] D. R. Smith, W. J. Padilla, D. C. Vier, S. C. Nemat-Nasser, and S. Schultz, Composite Medium with Simultaneously 
Negative Permeability and Permittivity, Phys. Rev. Lett. 84, 4184 (2000).

[14] M. Choi et al., A Terahertz Metamaterial with Unnaturally High Refractive Index, Nature (London) 470, 369 (2011).

[15] J. Valentine, S. Zhang, T. Zentgraf, E. Ulin-Avila, D. A. Genov, G. Bartal, and X. Zhang, Three-Dimensional Optical Metamaterial with a Negative Refractive Index, Nature (London) 455, 376 (2008).

[16] S. Sun et al., High-Efficiency Broadband Anomalous Reflection by Gradient Meta-Surfaces, Nano Lett. 12, 6223 (2012).

[17] X. Ni, N. K. Emani, A. V. Kildishev, A. Boltasseva, and V. M. Shalaev, Broadband Light Bending with Plasmonic Nanoantennas, Science 335, 427 (2012).

[18] S. Sun, Q. He, S. Xiao, Q. Xu, X. Li, and L. Zhou, GradientIndex Meta-Surfaces as a Bridge Linking Propagating Waves and Surface Waves, Nat. Mater. 11, 426 (2012).

[19] Y. Yang, W. Wang, P. Moitra, I. I. Kravchenko, D. P. Briggs, and J. Valentine, Dielectric Meta-Reflectarray for Broadband Linear Polarization Conversion and Optical Vortex Generation, Nano Lett. 14, 1394 (2014).

[20] B. Sensale-Rodriguez, R. Yan, M. M. Kelly, T. Fang, K. Tahy, W. S. Hwang, D. Jena, L. Liu, and H. G. Xing, Broadband Graphene Terahertz Modulators Enabled by Intraband Transitions, Nat. Commun. 3, 780 (2012).

[21] Y. Yao, R. Shankar, M. A. Kats, Y. Song, J. Kong, M. Loncar, and F. Capasso, Electrically Tunable Metasurface Perfect Absorbers for Ultrathin Mid-Infrared Optical Modulators, Nano Lett. 14, 6526 (2014).

[22] R. Degl'Innocenti, D. S. Jessop, Y. D. Shah, J. Sibik, J. A. Zeitler, P. R. Kidambi, S. Hofmann, H. E. Beere, and D. A. Ritchie Low-Bias Terahertz Amplitude Modulator Based on Split-Ring Resonators and Graphene, ACS Nano 8, 2548 (2014).

[23] B. Sensale-Rodriguez et al., Extraordinary Control of Terahertz Beam Reflectance in Graphene ElectroAbsorption Modulators, Nano Lett. 12, 4518 (2012).

[24] F. Wang, Y. Zhang, C. Tian, C. Girit, A. Zettl, M. Crommie, and Y.R. Shen, Gate-Variable Optical Transitions in Graphene, Science 320, 206 (2008).

[25] F. H. L. Koppens, D. E. Chang, and F. J. García de Abajo, Graphene Plasmonics: A Platform for Strong Light-Matter Interactions, Nano Lett. 11, 3370 (2011).

[26] L. Ju et al., Graphene Plasmonics for Tunable Terahertz. Metamaterials, Nat. Nanotechnol. 6, 630 (2011).

[27] I. Maeng, S. Lim, S. J. Chae, Y. H. Lee, H. Choi, and J.-H. Son, Gate-Controlled Nonlinear Conductivity of Dirac Fermion in Graphene Field-Effect Transistors Measured by Terahertz Time-Domain Spectroscopy, Nano Lett. 12, 551 (2012).

[28] N. K. Emani, T.-F. Chung, X. Ni, A. V. Kildishev, Y. P. Chen, and A. Boltasseva, Electrically Tunable Damping of Plasmonic Resonances with Graphene, Nano Lett. 12, 5202 (2012).

[29] J. Chen et al., Optical Nano-Imaging of Gate-Tunable Graphene Plasmons, Nature (London) 487, 77 (2012).

[30] S. H. Mousavi et al., Inductive Tuning of Fano-Resonant Metasurfaces Using Plasmonic Response of Graphene in the Mid-Infrared, Nano Lett. 13, 1111 (2013).

[31] H. Yan, X. Li, B. Chandra, G. Tulevski, Y. Wu, M. Freitag, W. Zhu, P. Avouris, and F. Xia, Tunable Infrared Plasmonic
Devices Using Graphene/Insulator Stacks, Nat. Nanotechnol. 7, 330 (2012).

[32] Z. Fang, Z. Liu, Y. Wang, P. M. Ajayan, P. Nordlander, and N. J. Halas, Graphene-Antenna Sandwich Photodetector, Nano Lett. 12, 3808 (2012).

[33] Z. Fang, S. Thongrattanasiri, A. Schlather, Z. Liu, L. Ma, Y. Wang, P. M. Ajayan, P. Nordlander, N. J. Halas, and F. Javier García de Abajo, Gated Tunability and Hybridization of Localized Plasmons in Nanostructured Graphene, ACS Nano 7, 2388 (2013).

[34] V. W. Brar, M. S. Jang, M. Sherrott, J. J. Lopez, and H. A. Atwater, Highly Confined Tunable Mid-Infrared Plasmonics in Graphene Nanoresonators, Nano Lett. 13, 2541 (2013).

[35] Z. Fei et al., Gate-Tuning of Graphene Plasmons Revealed by Infrared Nano-Imaging, Nature (London) 487, 82 (2012).

[36] F. Valmorra, G. Scalari, C. Maissen, W. Fu, C. Schönenberger, J. W. Choi, H. G. Park, M. Beck, and J. Faist, Low-Bias Active Control of Terahertz Waves by Coupling Large-Area CVD Graphene to a Terahertz. Metamaterial, Nano Lett. 13, 3193 (2013).

[37] S. H. Lee et al., Switching Terahertz Waves with GateControlled Active Graphene Metamaterials, Nat. Mater. 11, 936 (2012).

[38] R. Yan, B. Sensale-Rodriguez, L. Liu, D. Jena, and H. G. Xing, A New Class of Electrically Tunable Metamaterial Terahertz Modulators, Opt. Express 20, 28664 (2012).

[39] S. Fan, W. Suh, and J. D. Joannopoulos, Temporal Coupled-Mode Theory for the Fano Resonance in Optical Resonators, J. Opt. Soc. Am. A 20, 569 (2003).

[40] H.-T. Chen, W. J. Padilla, M. J. Cich, A. K. Azad, R. D. Averitt, and A.J. Taylor, A Metamaterial Solid-State Terahertz Phase Modulator, Nat. Photonics 3, 148 (2009).

[41] H.-T. Chen, W. J. Padilla, J. M. O. Zide, A. C. Gossard, A. J. Taylor, and R. D. Averitt, Active Terahertz Metamaterial Devices, Nature (London) 444, 597 (2006).

[42] D. Sievenpiper, Z. Lijun, R. F. J. Broas, N. G. Alexopolous, and E. Yablonovitch, High-Impedance Electromagnetic Surfaces with a Forbidden Frequency Band, IEEE Trans. Microwave Theory Tech. 47, 2059 (1999).

[43] J. M. Hao, L. Zhou, and C. T. Chan, An Effective-Medium Model for High-Impedance Surfaces, Appl. Phys. A 87, 281 (2007).

[44] See Supplemental Material at http://link.aps.org/ supplemental/10.1103/PhysRevX.5.041027 for sample preparation and characterization, ion-gel gating technique, experimental data at hole doping, absolute reflectance and operating efficiency of the devices, and possible methods to further improve the range of phase modulation.

[45] A. Silva, F. Monticone, G. Castaldi, V. Galdi, A. Alù, and N. Engheta, Performing Mathematical Operations with Metamaterials, Science 343, 160 (2014).

[46] A. Pors, M. G. Nielsen, and S. I. Bozhevolnyi, Analog Computing Using Reflective Plasmonic Metasurfaces, Nano Lett. 15, 791 (2015).

[47] H. Tao, N. I. Landy, C. M. Bingham, X. Zhang, R. D. Averitt, and W. J. Padilla, A Metamaterial Absorber for the Terahertz Regime: Design, Fabrication and Characterization, Opt. Express 16, 7181 (2008). 
[48] J. Hao, J. Wang, X. Liu, W. J. Padilla, L. Zhou, and M. Qiu, High Performance Optical Absorber Based on a Plasmonic Metamaterial, Appl. Phys. Lett. 96, 251104 (2010).

[49] E. H. Hwang and S. Das Sarma, Dielectric Function, Screening, and Plasmons in Two-Dimensional Graphene, Phys. Rev. B 75, 205418 (2007).

[50] K. Ding, Y. Shen, J. Ng, and L. Zhou, Equivalent-Medium Theory for Metamaterials Made by Planar Electronic Materials, Europhys. Lett. 102, 28005 (2013).
[51] R. Serway and J. Jewett, Principles of Physics: A CalculusBased Text (Cengage Learning, Boston, 2005).

[52] N. Laman and D. Grischkowsky, Terahertz Conductivity of Thin Metal Films, Appl. Phys. Lett. 93, 051105 (2008).

[53] S. Xiao, Q. He, X. Huang, S. Tang, and L. Zhou, Enhancement of Light-Matter Interactions in Slow-Wave Metasurfaces, Phys. Rev. B 85, 085125 (2012). 